\title{
Pancreatoblastoma, a Rare Childhood Tumor: A Case Report
}

\author{
Asuman ARGON', Ahmet ÇELIK ${ }^{2}$, Haldun ÖNiZ ${ }^{3}$, Geylani ÖZOK², Funda YILMAZ BARBET ${ }^{4}$ \\ 'Department of Pathology, İmir Bozyaka Training and Research Hospital, iZMiR, TURKEY \\ Department of ${ }^{2}$ Pediatric Surgery and ${ }^{4}$ Pathology, Ege University, Faculty of Medicine, IZMIR, TURKEY \\ ${ }^{3}$ Clinic of Pediatric Oncology and Bone Marrow Transplantation, Izmir Tepecik Training and Research Hospital, IZMiR, TURKEY
}

\begin{abstract}
Pancreatoblastoma, rarely encountered in the literature, is a malignant exocrine tumor seen in the pancreas. A 5-year-old boy suffering from abdominal pain was sent to our institute for further examination and treatment. Clinical examination was normal but for a palpable abdominal tumor mass. Abdominal Doppler ultrasonography showed a mass with well-defined margins within the body of the pancreas. Laboratory tests, including lactic dehydrogenase, alpha-fetoprotein and cancer antigen 125 were abnormal. The tumor invading the splenic vein and transverse colon was removed totally. We observed a hypercellular tumor in histopathological examination. The tumor had epithelial acinar cells and squamoid morules (corpuscles) separated by stromal bands. Adjuvant chemotherapy was used after surgery. However, the patient died 14 months later. All data about pancreatoblastoma have to be collected in order to choose the treatment to elucidate the molecular pathogenesis of the tumor, to diagnose it early and to develop target-specific treatments.
\end{abstract}

Key Words: Child, Pancreatic neoplasms, Pancreatoblastoma

\section{INTRODUCTION}

In childhood, exocrine pancreatic tumors are unusual diseases (1). Recently, there is a growing interest in these unusual tumors along with the advances in the therapeutic concepts. Pancreatoblastoma (PB) arises in pancreas and is reported with an annual incidence of around 0.004 new case per 100,000 population (2). PB is a rare tumor; nevertheless, it is among the most common malignant neoplasms in pancreas (3). PB is frequently seen in childhood, but it may occur at any stage of life; nevertheless, adults and fetus appear to be free from this disease $(4,5)$. PB is seen more often in females than males (3). Although this tumor was formerly known as "infantile carcinoma of the pancreas", Horie et al. (6) named the tumor PB in 1977. PB is composed of squamoid corpuscles and acinar cells with zymogen granules and these cells form an organoid pattern containing lobular structures (1). The above-mentioned appearance resembles fetal pancreatic tissue. Alphafetoprotein (AFP) is the tumor marker used most often in PB (7). The biological behavior of PB in children is less aggressive but local invasion, recurrence, and metastasis are frequently seen (8). Although the surgical approach for PB is not standardized, total resection is so far the only choice for treating these patients (9).

(Turk Patoloji Derg 2017, 33:164-167)

Received : 22.03.2013 Accepted : 21.06.2013

\section{CASE REPORT}

A 5-year-old boy suffering from abdominal pain was referred to our institute for further examination and treatment. Three days earlier, he had been examined for abdominal pain elsewhere and abdominal ultrasonography (US) had identified a solid heterogeneous mass measuring $11 \mathrm{~cm}$ in diameter close to the left kidney, together with three solid hypoechoic metastatic masses measuring $28 \mathrm{~mm}, 25 \mathrm{~mm}$ and $15 \mathrm{~mm}$ in the liver left lobe, respectively. The initial diagnosis was germ cell tumor. Clinical examination was normal in our hospital except for a palpable abdominal tumor mass. He had no co-morbidities and his medical history revealed nothing of significance. Laboratory tests showed that hemoglobin was $9.4 \mathrm{ng} / \mathrm{ml}$, hematocrit $28 \%$, and lactic dehydrogenase $833 \mathrm{ng} / \mathrm{ml}$. Abnormal tumor markers levels including alpha feto protein (AFP) of $55117 \mathrm{ng} / \mathrm{ml}$, and carcino embryonic antigen $125835 \mathrm{ng} / \mathrm{ml}$ were present while human chorionic gonadotropin-beta was normal. Abdominal Doppler US showed a mass in the body of the pancreas which had well-defined margins and measured $11 \times 8 \mathrm{~cm}$. The tumor was solid but it also contained a hyper echoic component. Computed tomography identified a solid heterogeneous mass measuring $11 \times 10 \times 8 \mathrm{~cm}$ in diameter (Figure 1).
Correspondence: Asuman ARGON

İzmir Bozyaka Eğitim ve Araştırma Hastanesi, Tıbbi Patoloji Kliniği,

35300 Karabağlar, İZMİR, TURKEY

E-mail: asumanargon@gmail.com Phone: +90 2322505050 
The tumor invading the splenic vein and transverse colon was removed totally (Figure 2). On gross examination, the perforated capsule surrounding the tumor was seen. The tumor measured $12.5 \times 12 \times 6 \mathrm{~cm}$. On cut section there was hemorrhage and also necrosis. We observed a hypercellular tumor in the histopathological examination. The tumor had cell clusters separated by stromal bands and composed of squamoid morules (corpuscles) and epithelial acinar cells. An increase in the nucleus/cytoplasm ratio and nucleomegaly were seen in the epithelial cells that had a pancreatic acini-like pattern. Although the nuclear membranes of the neoplastic cells were regular, coarse chromatin and multiple conspicuous nucleoli were

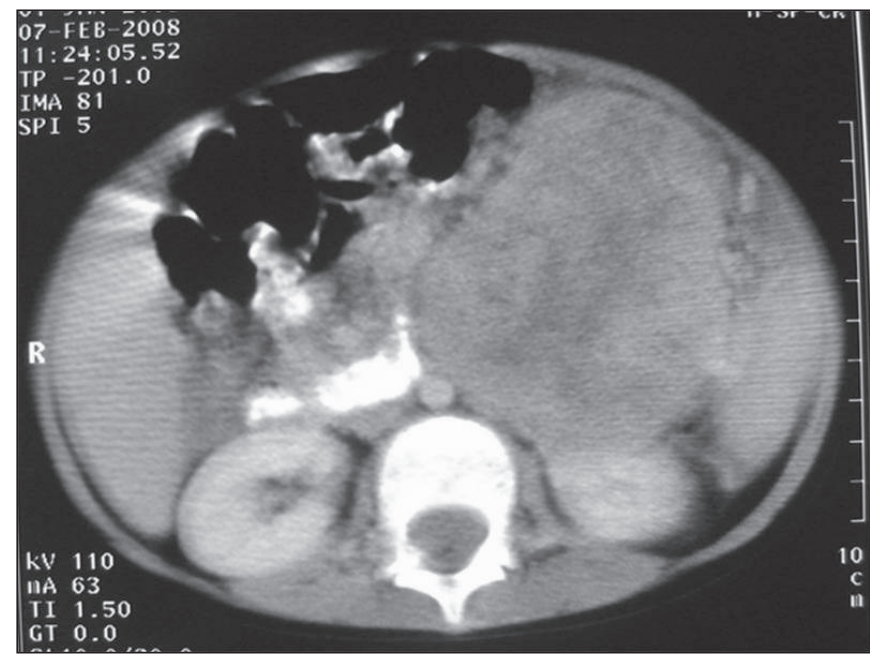

Figure 1: Computed tomography identified a solid heterogeneous mass measuring $11 \times 10 \times 8 \mathrm{~cm}$ in diameter close to the left kidney.

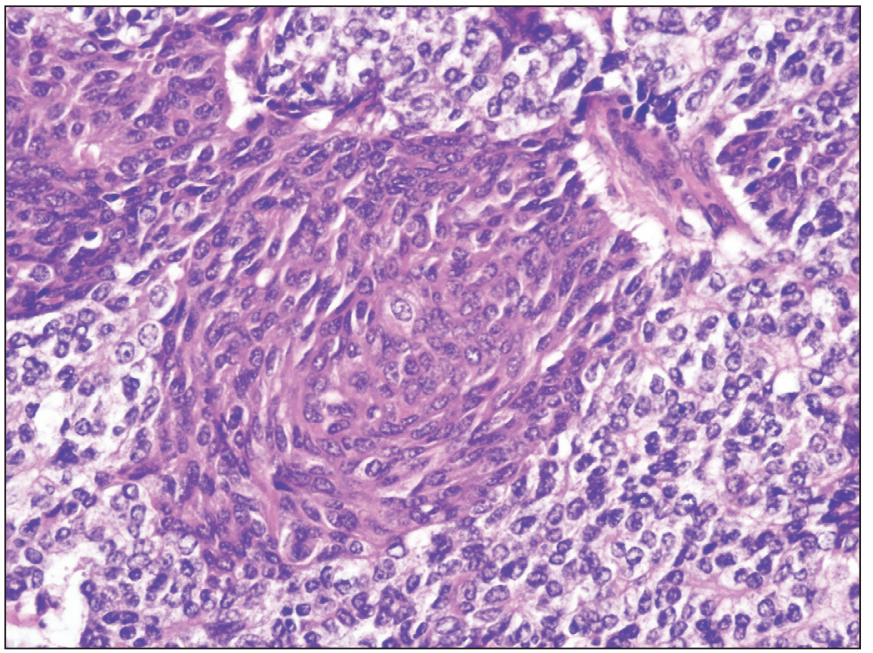

Figure 3: Squamoid corpuscles were seen in many clusters of tumor cells. In the squamoid corpuscles, epithelioid cells were plump and also had a dense eosinophilic cytoplasm (H\&E; x20). observed and the cells had a pale eosinophilic cytoplasm. Squamoid corpuscles were seen in many clusters of tumor cells. In the squamoid corpuscles, the epithelioid cells were plump and contained dense eosinophilic cytoplasm (Figure 3). Immunohistochemically, the neoplastic cells were positive with alpha-1-antitrypsin but showed no staining with WT-1, monoclonal carcinoembryogenic antigen or Tag72. CD10 and fascin were positive in squamoid corpuscle cells. Nuclear and strong cytoplasmic staining for ß-catenin (Figure 4) was seen in squamoid corpuscle cells but non-neoplastic pancreatic acini showed the expected membranous and faint cytoplasmic staining. In addition, chromogranin A and synaptophysin showed positivity

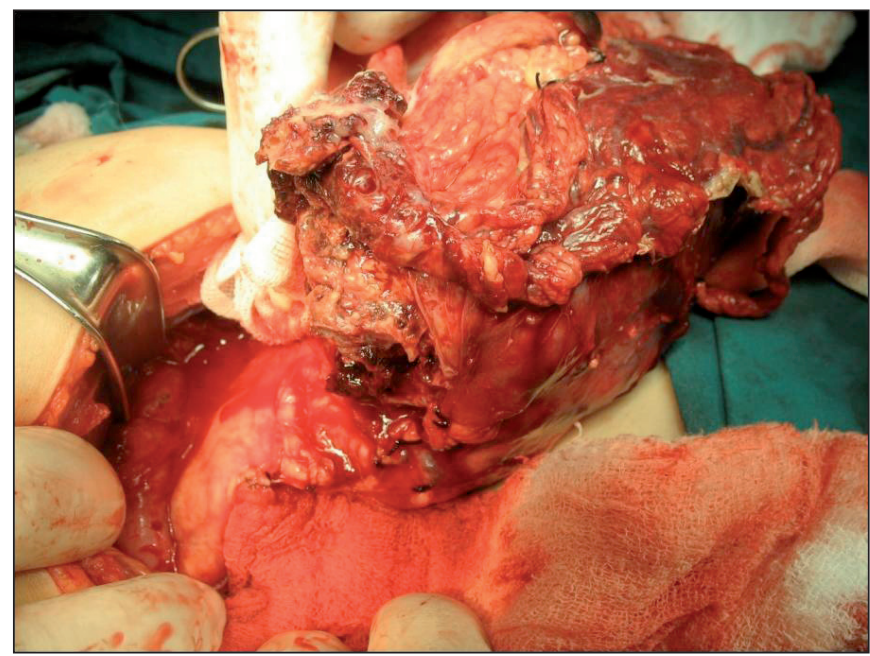

Figure 2: The tumor invading the splenic vein and transverse colon was totally removed. Gross pathologic examination showed an encapsulated tumor and measuring $12.5 \times 12 \times 6 \mathrm{~cm}$ in diameter.

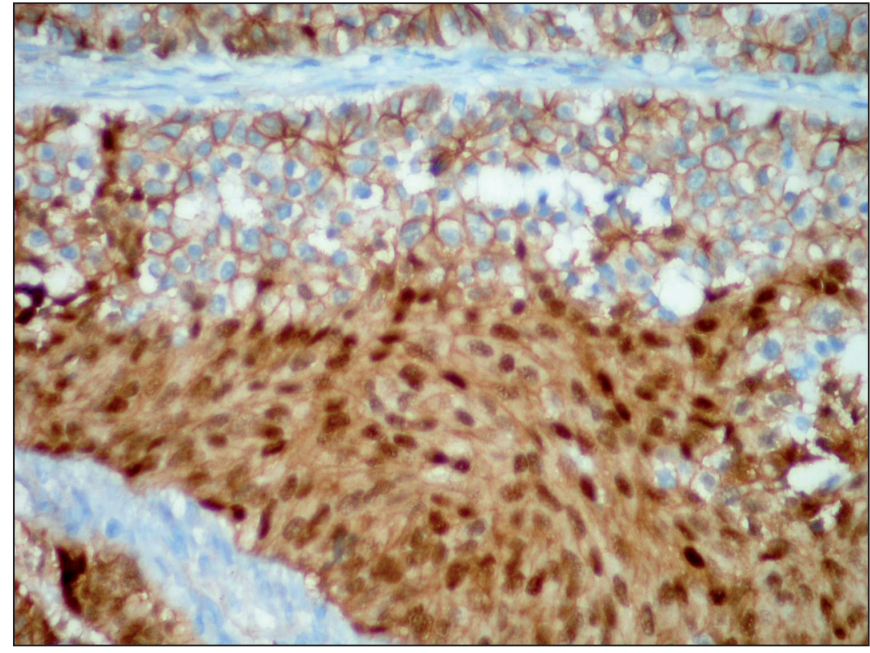

Figure 4: Immunohistochemically, nuclear and cytoplasmic staining were seen in the neoplastic cells for $ß$-catenin ( $ß$-catenin; $\mathrm{x} 20$ ). 
in a few endocrine cells. Typical histological features and immunohistochemistry were suggestive of the diagnosis of PB. After surgery, AFP levels decreased to $72.6 \mathrm{IU} /$ $\mathrm{ml}$ gradually. Adjuvant chemotherapy was used as of the 14th day after surgery. The chemotherapy regimen was as follows: every fifteen days; cisplatin $80 \mathrm{mg} / \mathrm{m} 2,24$ hours constant infusion; carboplatin $500 \mathrm{mg} / \mathrm{m} 2$, 1-hour infusion and doxorubicin $60 \mathrm{mg} / \mathrm{m} 2$, 48 hours constant infusion. On the postoperative 43rd day, metastatic lesions in the liver were stable and there was a recurrent mass in the pancreas region. With these findings, we concluded that the disease had progressed. The chemotherapy regimen was changed to carboplatin $400 \mathrm{mg} / \mathrm{m} 2 /$ day, intravenous, 1-day infusion for 2 days; ifosfamide $1800 \mathrm{mg} / \mathrm{m} 2 /$ day, intravenous, 1-day infusion for 5 days; etoposide $100 \mathrm{mg} / \mathrm{m} 2 /$ day, intravenous, 1-day infusion for 5 days. The radiological evaluation after 6th chemotherapy regimen revealed that the recurrent mass in the pancreatic region had disappeared and metastatic lesions in the liver had regressed. However, an operation was not considered in the case of this patient as the metastatic lesions in the liver were multiple and unresectable. The chemotherapy was therefore continued. Severe neutropenia and progression in the metastatic lesions in the liver were detected during the evaluation following the 9th chemotherapy regimen, The treatment was discontinued upon the request of the parents. The patient died on the 14th month following the operation.

\section{DISCUSSION}

Pancreatoblastoma (PB) is an unusual malignant exocrine tumor arising in pancreas (1). There have been approximately 200 cases in literature since Becker described PB in 1957 (3). $\mathrm{PB}$ is frequently seen in Asians, females and childhood as in our case (10). It may stem from any part of the pancreas. This tumor was divided into two categories by Horie et al. (6) as right-side tumors and left-side tumors. According to Horie et al. (6), the right-side tumors arise from the primordial ventral pancreas, do not contain calcification and islet cells, are usually well-encapsulated and have a better prognosis. However, the left-side tumors arise from the primordial dorsal pancreas, contain calcification and islet cells, have no encapsulation, and indicate a poorer prognosis than right-side tumors $(6,11)$. The tumor in our patient has the features of a left-side tumor because it arose from the body of pancreas, contained islet cells and had a poor prognosis. However it also had the features of a right side tumor as it was well-encapsulated and did not show calcification. Larger series are needed in order to understand the clinical and prognostic significance of the tumors that have mixed features as in our case.
As already known, both the pancreas and the liver stem from the same primitive cells (12). Therefore, the fact that serum AFP levels are increased in PB cases is not surprising, as in hepatoblastoma (7). Some authors reported a loss of heterozygosity $(\mathrm{LOH})$ on chromosome 11 in PBs $(13,14)$. According to Kerr et al. (14), although extensive LOH of the chromosome 11 encompassed the WT-1 gene, mutations were not detected in WT1 gene exons. Abraham et al. (13) observed somatic mutations in the APC/ $B$-catenin pathway but they did not detect any changes in the p53 and K-ras genes. Also, they postulated that a significant correlation was detected between immunohistochemical ß-catenin positivity and the APC/ $\beta$-catenin gene alterations (13). The molecular and immunohistochemical studies suggest that most PBs exhibit typical genetic alterations and they are similar to other embryonal tumors such as acinar cell carcinoma and hepatoblastoma (12-15). In our case, immunohistochemically, ß-catenin positivity and WT-1 negativity resemble the data in the literature. Molecular studies in large series may help elucidate the pathogenesis of $\mathrm{PB}$.

The radiological differential diagnosis includes pancreatic cystic neoplasm, ductal adenocarcinoma, acinar cell carcinoma, nonfunctioning endocrine pancreatic tumors, solid pseudo papillary tumors, peripancreatic and pancreatic lesions, especially tuberculosis and autoimmune pancreatitis $(4,16)$. In early childhood, the spectrum of differential diagnosis is wide when it cannot be clearly seen that the tumor arises from the pancreas (11). The tumors that must be considered first are Wilms tumor, neuroblastoma, hepatoblastoma, and other primary liver tumors $(12,13)$. Acinar cell carcinoma and PB have similar histopathological and radiologic appearances $(1,11)$. However, PB usually occurs in the first decade of childhood, whereas acinar carcinoma is encountered in older patients. Moreover, squamoid corpuscles are typical in PB but they do not exist in acinar cell carcinoma $(1,12)$.

Clinicopathologic features, treatment and prognosis of $\mathrm{PB}$ were evaluated in the paper by Dhebri et al. (17). They reported that $78 \%$ of the 153 patients were under 10 years. The symptoms and signs were like those described in other articles $(10,18)$. Moreover, $17 \%$ of their patients had metastatic disease at the time of diagnosis. The study of Dhebri et al. (17) showed that the 5-year survival rate of patients after total resection was $65 \%$ whereas median survival of their patients with non resectable disease at the time of diagnosis was 36 months. Furthermore, all of these patients died before 40 months. According to their study, the best choice of treatment is complete resection. If 
the tumor is unresectable, $\mathrm{PB}$ is treated with chemotherapy $(17,19)$. Although vincristine, doxorubicin, etoposide, cyclophosphamide, cisplatin and adriamycin have turned out to be effective chemotherapeutic agents, there are no established or proven chemotherapy regimens (19). The efficiency of neoadjuvant chemotherapy was not clearly proven in any studies (17). New studies with larger series are still needed. Our patient underwent chemotherapy that contained two different groups of medicine for 12 cycles. The first group contained carboplatin, doxorubicin and cisplatin and early relapse and progression were detected during this treatment. The second group contained ifosfamide, carboplatin and etoposide. Regression was found during the 6 courses of chemotherapy but the disease then progressed. Data on the basis of similar cases are needed in order to determine the alternative treatment options and the efficiency of the chemotherapy treatments.

According to SEER data (1973-2004), median survival in $\mathrm{PB}$ is 193 months and the 15 -year survival rate is $75 \%$ but the initial tumor stage was not reported in this study (2). Our patient had metastatic disease at the time of diagnosis. Although the primary tumor was completely resected in our patient, metastatic lesions in the liver could not be resected. While the first chemotherapy regime was inefficient, response to the second chemotherapy regimen was limited. When these findings were assessed together with literature, early diagnosis and complete resection of the tumors seem to be important in order to decrease the tumor load and to increase the response to treatment.

All data about PB have to be collected in order to choose the treatment to elucidate the molecular pathogenesis of the tumor, to enable early diagnosis, and to develop targetspecific treatments.

\section{REFERENCES}

1. Tumors of the Pancreas. In: Hruban, RH, Pitman MB, Klimstra DS, editors. AFIP Atlas of Tumor Pathology Series 4. Washington DC: ARP Press; 2007.111-64.

2. National Cancer Institute Surveillance, Epidemiology and End Results (SEER) Program Public-Use Data 1973- 2005. National Cancer Institute, DCCPS, Surveillance Resaerch Program, Cancer Statistics Branch, Based on the November 2007 submission[cited 2008 April]. Available at: www.seer.cancer.gov.

3. Perez EA, Gutierrez JC, Koniaris LG, Neville HL, Thompson WR, Sola JE. Malignant pancreatic tumors: Incidence and outcome in 58 pediatric patients. J Pediatr Surg. 2009;44: 197-203.
4. Savastano S, d'Amore ES, Zuccarotto D, Banzato O, Beghetto M, Famengo B. Pancreatoblastoma in an adult patient. A case report. JOP. 2009;10:192-5.

5. Chisholm KM, Hsu CH, Kim MJ, Rangaswami A, Gray Hazard FK. Congenital pancreatoblastoma: Report of an atypical case and review of the literature. J Pediatr Hematol Oncol. 2012;34:310-5.

6. Horie A, Yano Y, Kotoo Y, Miwa A. Morphogenesis of pancreatoblastoma, infantile carcinoma of the pancreas: Report of two cases. Cancer. 1977;39:247-54.

7. Morohoshi T, Sagawa F, Mitsuya T. Pancreatoblastoma with marked elevation of serum alpha-fetoprotein. An autopsy case report with immunocytochemical study. Virchows Arch A Pathol Anat Histopathol. 1990;416:265-70.

8. Lee YJ, Hah JO. Long-term survival pancreatoblastoma in children. J Pediatr Hematol Oncol. 2007;29:845-7.

9. Dumitrascu T, Stanciulea O, Herlea V, Tomulescu V, Ionescu M. Central pancreatectomy for pancreatoblastoma in a 16-year-old girl. J Pediatric Surg. 2011;46:17-21.

10. Gu WZ, Zou CC, Zhao ZY, Liang L, Tang HF. Childhood pancreatoblastoma: Clinical features and immunohistochemistry analysis. Cancer Lett. 2008;264:119-26.

11. Herman TE, Siegel MJ, Dehner LP. CT of pancreatoblastoma derived from the dorsal pancreatic anlage. J Comput Assist Tomogr. 1994;18:648-50.

12. Saif MW. Pancreatoblastoma. JOP(Online). 2007;8:55-63.

13. Abraham SC, Wu TT, Klimstra DS, Finn LS, Lee JH, Yeo CJ, Cameron JL, Hruban RH. Distinctive molecular genetic alterations in sporadic and familial adenomatous polyposisassociated pancreatoblastoma: Frequent alterations in the APC $/ \beta$-catenin pathway and chromosome $11 \mathrm{p}$. Am J Pathol . 2001;59:1619-27.

14. Kerr NJ, Chun YH, Yun K, Heathcott RW, Reeve AE, Sullivan MJ. Pancreatoblastoma is associated with chromosome $11 \mathrm{p}$ loss of heterozygosity and IGF2 overexpression. Med Pediatr Oncol. 2002;39:52-4.

15. Klimstra DS. Nonductal neoplasms of the pancreas. Mod Pathol. 2007;20:94-112

16. Cavallini A, Falconi M, Bortesi L, Crippa S, Barugola G, Butturini G. Pancreatoblastoma in adults: A review of the literature. Pancreatology. 2009;9:73-80.

17. Dhebri AR, Connor S, Campbell F, Ghaneh P, Sutton R, Neoptolemos JP. Diagnosis, treatment and outcome of pancreatoblastoma. Pancreatology. 2004;4:441-53.

18. Rich RH, Weber JL, Shandling B. Adenocarcinoma of the pancreas in a neonate managed by pancreatoduodenectomy. J Pediatr Surg. 1986;21:806-8.

19. Vossen S, Goretzki PE, Goebel U, Willnow U. Therapeutic management of rare malignant pancreatic tumors in children. World J Surg. 1998;22:879-82. 